\title{
Literature Review of Facial Skin Embellishment Using Flexible Region Aware Mask
}

\author{
Priyanka Mehra ${ }^{1}$, A. S. Deshpande ${ }^{2}$ \\ ${ }^{1,2}$ E \& TC, ICOER Pune, India
}

\begin{abstract}
Facial attractiveness enhancement is becoming more and more important for beautification. So I am proposing flexible region aware mask technique for three major skin attributes: homogeneity, lighting, and color. For implementing such specific facial attractiveness, there is a new region-aware mask generation technique which is based on the edit propagation techniques. The regionaware masks allow the user to beautify faces. This paper propose an input image which is decomposed into three different layer by using edge-preserving filter. After decomposing the facial landmarks and significant edges as the restraint, layer masks which are generated based on the guided image of energy minimization technique. Our method could beautify faces automatically using the guided image. The result which has come based on the various databases.
\end{abstract}

Keywords: Facial skin embellishment, Flexible region aware mask, Edit propagation technique, Facial attractiveness

\section{Introduction}

People have always sought out beauty and countless philosophers, artists, and scientists have tried to capture the nature of beauty. Facial attractiveness has been studied extensively as an important part of aesthetics, and recent is consistent among individuals, regardless of their gender, ethnicity, or age.A universal definition of beauty remains ambiguous,but the consistent perception of facial attractiveness indicates that machine based analysis of facial beauty and facial beautification will probably have many useful applications, as well as role in research.

Face makeup which is used for a beautification purpose is to change an appearance using special cosmetics such asfoundation, powder, cream etc. Many times females do make up to enhance her beauty using foundation and loose powder which are usually used to change the texture of face's skin. Foundation is mainly used to cover up her defect and skin texture, while the loose powder is used for looking pleasant.

Afterwards, some other color makeup, such as rouge, eye liner and shadow, etc. which used on top of the powder.

When a female enters a beauty salon, she selects image from a catalog and tells the beautician to apply the same makeup on her. Before going to actual task, it would be helpful.Traditionally, the female have two choices for trying out makeup. One is to physically apply the makeup, whichistime consuming and requires the patience. Alternatively, she can try digitally makeup by digital photography and with the help of photo editing software, such as Adobe Photoshop ${ }^{\mathrm{TM}}$. But using such photo editing software it takes to much effort on such a tedious work.

In this paper our approach is based on physical method. First, wedecompose the guided image (subject) and example image into three layers separately: intensity layer, skin detail layer, and color layer[34]-[36].basically the intensity layer contains eyes, nose, mouth, etc. The skin detail layer contains the skin texture, including dark circles, wrinkles etc. The color layer represents the smoothness of skin. After the three layers are decomposed, face makeup by guided image can be considered as transferring the skin detail layer and color Layer from the makeup are based on the subject image while preserving the face structure layer of the subject image.

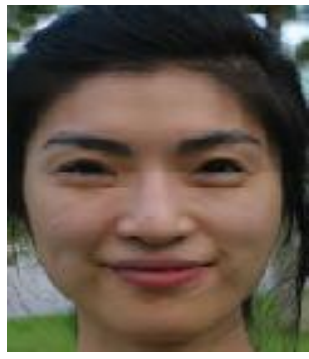

(a)

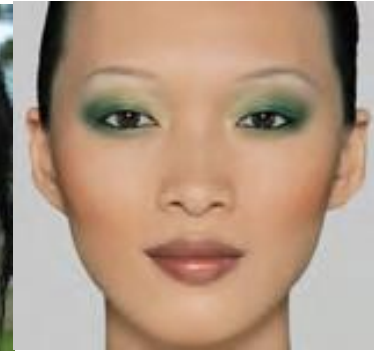

(b)

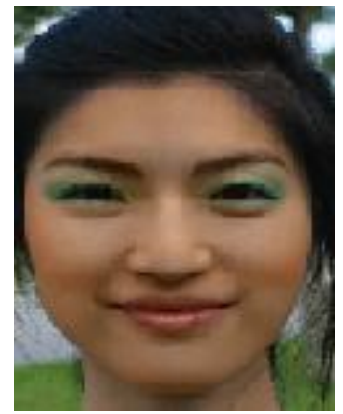

(c)

Figure 1: Face makeup by example. (a) A subject image (b) An example style image, [11]. (c) The result of our approach,

\section{Literature Review}

Face beautification is generally used for an Advertisements, magazines and facebook, which manipulateplentiful facial images every day. The image -editing software system are available (such as Adobe Photoshop ) is more popular for face beautification but some modification is still take some time. As well as social network has become more popular for image enhancement. Mostly the users requirement is immediately do facial beautification with the minimum number of operations to avoid endless manipulations. There 


\section{International Journal of Science and Research (IJSR) \\ ISSN (Online): 2319-7064 \\ Index Copernicus Value (2013): 6.14 | Impact Factor (2015): 6.391}

for it is useful to develop a face image enhancement technique that is effective ,convenient, and flexible.The increasing demand for face image retouching has worked on many methods,such as facial geometric beautification [34],digital facial makeup[29], personal photo enhancement[20], and hair modeling[21].

In the last few years facial skin beautification [24]-[26] has become one of the most important and time consuming tasks during face image editing. When facial skin is manipulated the editied regions should be selected accurately by a facial mask to avoid visual artifacts.it is possible to draw (or paint) mask manually, but this involves many complex operations. According to the recent research, there are two ways of obtaining facial mask. The first method regards facial mask generation as a specific image segmentation problem, which involves integrating the facial priors into a segmentation model. Another method called asan edit propagation technique has been proposed [37], to achieve this goal, in which user frequently propagates the entire image according to thepixel affinity .

The first step in all the faces contained in the images are located, along with their key features. first it generate a bounding box around each faces. To extract human face from given image, many algorithms have been proposed. Skin color detection algorithms proposed to extract the skin parts from image.Most of these approaches use statistical method to find out the boundry of human skin color space.There are some requirements of a skin color model.

\subsection{Facial Beautification}

Blanz and Vetter [22], [23] to generate attractive face image. In facial beautification facial geometry[34], is one of the important facial attributes that affects attractiveness and it has been studied widely to generate attractive facse using 3D morphable models ,

Eisenth et al. [33] to generate attractiveness, it predicted attractiveness using machine-learning methods, including Knearest neighbor algorithm and support vector regression.For facial attractiveness three major attributes that influence smoothness, lighting, and color .

For Facial skin manipulation Lee et al. [26] generated generated a skin seagmentation map using a Gaussian mixture model and Bayesian segmentation to allow automatic skin smoothness enhancement. Chen et al. [25] performed automatic skin color enhancement based on color temperature-insensitive skin color detection and editied faces using a bilateral filter with poisson image cloning.

During facial beautification (or even general image editing),one of the key stages is to isolate the editing regions and determine the relative adjustment level between these regions. It accomplish both taskssimultaneously using a layer mask. However, general mask painting is a time-consuming and tedious task.It looks attractive if produce the mask automatically, but mask generation is very challenging due to the high facial variances in the real-world condition.
There is not much previous work addressing digital face makeup. The most closely related work is that of Tong etal. [15].is to change the appearance which is learned from a pair of sample image of the same image "before" and "after" makeup. The out come of the result "after" splitting of the image which is used "before". Then the result which has come is multiplied by another image to achieve the better result. In contrast, our approach requires only one "after" subject image. This is more flexible and practical, as providing the "before" image is rather difficult in most cases. the makeup is used to change the texture of face skin. Because the original texture varies from person to person, After makeup it shows the changes for different faces. Thus, it is incorrect to apply the change across two faces.

Another approach by Ojima et al. [10]is used a pair of "bereaved" makeup . However, only the foundation effect is addressed in their work. In contrast,Tsumura et al. [16] is worked on physical model to extract vital fluid and melanin components. Changes in facial appearance are simulated by adjusting the amount of vital fluid and melanin. The effects they demonstrated includetanning, reddening due to alcohol consumption, aging and cosmetic effects. However, the cosmetic effects are quite limited, and are much simpler than real makeup. Besides,an online commercial software, Taaz [13], provides users with virtual makeup on face by simulating the effects of specified cosmetics,

Furthermore some existing works also focus on the beautification of face photos. It proposed an automated face photo retouching method aiming to detect and remove spot, beauty mark, and acne from faces. Another technique is used to implement the face structure and to enhance the beauty. However, this may also change the identity of the image, as face structure is normally considered as a key to represent as an identity. similarly, we achieve the goal of beautification by changing the skin detail layer and color, while faithfully preserving the face structure.

The idea of image processing by example can be found in image [5]. Image analogies provide a general framework to classified an image in different styles. This technique is used to learns how pixel values change from a pair of "be-reved" images as example. earlier, we saw the difference is that our approach learns the effects after alteration, while their approach learns the way of altering image pixels.

Shan et al. [12] proposed method to transfer the texture across two images. Their method of transferring fine texture is similar to ours in spirit. They used the quotient of the original image and a Gaussian smoothed version of the image to represent the texture. The quotient is multiplied to the smoothed version of another image. This is similar to our layer decomposition. However, there are many differences. First, the Gaussian blur they used may produce halo effect at the strong edges. In contrast,edge preservingsmooth [9] used to separate the layers, which successfully suppresses the halo effects. Moreover, they only focus on texture transfer; but it is also important to keep color consistent. We separately transfer the skin detail in lightness channel and color in color channel. In addition, they focused on transferring texture, while our goal is to transfer makeup effect, which is more complex. 


\section{International Journal of Science and Research (IJSR) \\ ISSN (Online): 2319-7064 \\ Index Copernicus Value (2013): 6.14 | Impact Factor (2015): 6.391}

\section{Methodology}

The preprocessing step in all the faces consist of the images are located, along with their basic features. This is done by first generating a bounding box around each face, later on it identifying the location of the chin, nose, mouth, eyes, and eyebrows of each person. As a rough figure is sufficient for our purpose, a polygonal model is used to represent the feature figure.

Face detection is proficient using a multi-view face detector based on the Viola et al. [4], which depend on cascading classifiers in such a way that the classifers in the early stage quickly discard simple non-face regions and in the latter stage it has designed to classify more complex cases. Using AdaBoost algorithm Each classifer is made up of a set of weak binary classifers, which are learned from a training set.

In this paper to extract face from given image, many algorithms have been proposed. Viola et al. proposed an efficient face detection technique [4]. There are three main principles in their algorithm, including the integral image, Ada boost, and cascade classifier. Later on Lienhart et al. proposed Haar-like features for object detection [2]. In this paper apply Lienhart's frontal faceclassifier [6] and Hameed's eye classifier [7] to extract human face and human eyes from given image respectively.

The objective of human eye detection is to subtract the effect of non-skin tone Element from detected human face. The result of human face detection and human eye detection. The workflow of our approach is illustrated that there are four main steps. First, face alignment should be done between the subject and guided images. Because the information is transferred pixel by pixel, a fully alignment is necessary before transferring. Followed is layer decomposition. It can be decomposed into three layers: face structure layer, skin detail layer, and the color layer. Third, information from each layer is transferred to corresponding layer in different fashions: skin detail is transferred in an additive way color is transferred by alpha blending highlight and shading effects in face structure layer are transferred in the way of gradient editing Finally, three resultant layers are composed together.

\subsection{Face Alignment}

For face alignment, the method which is used called as Thin Plate Spline (TPS) [1] to warp the guided image to subject image. The control points required by TPS are obtained using an extended Active Shape Model (ASM) [8] .

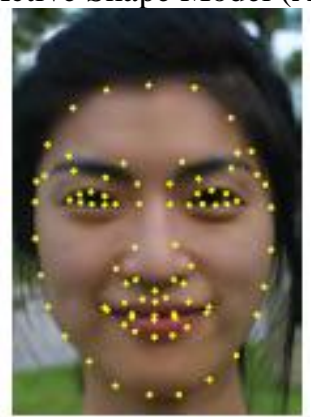

(a)

Due to the dissimilarity of face appearance under various possible make up, ASM may not get the accurate position of points. Our proposed method may still require user to refine the position of control points. Since the control points have been roughly located already, the refinement does not require much effort. It usually takes less than 1 minute to refine the control points for a face.

The control points have defined different face components, viz eyebrows, eyes, nose, nostrils, lips, mouth cavity (the space between lips), and other facial skin (the rest of the face). These components are further divided into three classes to be treated in different ways during makeup. These three classes (C1 C3) are illustrated in different colors. C1 (the skin region, the entire face excluding $\mathrm{C} 2$ and $\mathrm{C} 3$ ) follows the work flow Since the texture of C2 (lips) varies greatly from person to person and the region of C2 is easy to deform, we use a special method to transfer the makeup style in this region . C3 (eyes and mouth cavity) is kept untouched all the time during entire makeup process.

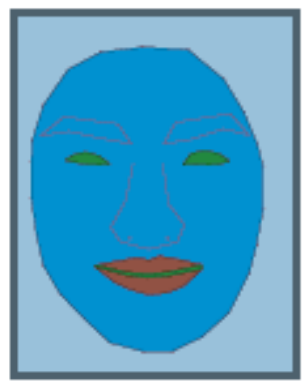

(b)

Figure 2: (a) Control points on a face. (b) Facial components

\subsection{Layer Decomposition}

The subject image and guided image (after warping) are first decomposed into color and lightness layers.We then further decompose the lightness layer into face structure and skin detail layers.

Large-scale/detail layer decomposition has been done in many layers, such as [3] and [19]. The main idea is first to perform an edge-preserving smoothing operator on the luminance layer to obtain the large scale layer, and then to subtract (or divide) the large scale layer from the lightness layer to obtain the detail layer. This approach,is based on weighted least-squares (WLS) operator recently proposed by Farbman et al. [9]. An alternative method is bilateral filtering[14], which was used in many previous works. We choose this WLS operator because of its better perform compared to the bilateral filter, especially when the blur level increases.

\subsection{Feature Extraction}

The goal of the feature extraction is to locate significant facial regions, which are used as the constraints for mask generation the important facial features include facial components.(eg, eyes, nose and mouth),details (such as wrinkles caused by expressions),textures (eg,beards and hair), and accessories (such as glasses).

These all features detector could be integrate into the frame work, but such implementation is not so easy.by analyzing 


\section{International Journal of Science and Research (IJSR) \\ ISSN (Online): 2319-7064 \\ Index Copernicus Value (2013): 6.14 | Impact Factor (2015): 6.391}

these features in this paper it include facial components and regions with meaningful facial attributes.

\subsection{Adaptive region aware mask generation}

The proposed method which is used to remove the unwanted details (such as spots) as well as adjust the facial attributes (such as skin color) in certain region without changing information in other regions (such as background).

To avoid the tedious mask ,proposed method region aware mask using the adaptive edit propagation technique.In this it extracted facial features which is treated as an input data. The pixel value of this data are propagated adaptively.

\section{Conclusion}

A facial enhancement scheme suggested to beautify face by using adaptive edit propagation technique, which enhance the images using different features detector or called as an operator .Here we use the different features detector which decompose the layers into three parts in an effective manner. The layer which is used to enhance the features it is digital image which edit separate different elements of an image. further we use the GUI System that help automatic and interactive facial skin beautification.

\section{References}

[1] F. Bookstein. Principal warps: Thin-plate splines and the decomposition of deformations. IEEE Trans. Pattern Analysis and Machine Intelligence, 11(6):567-585, 1989.

[2] Lienhart, R., Maydt, J.: An extended set of Haar-like features for rapid object detection. In:Proceedings of 2002 International Conference on Image Processing, vol. 901, pp. I-900-I-903 (2002)

[3] E. Eisemann and F. Durand. Flash photography enhancement via intrinsic relighting. ACM Trans. Graphics, 23(3):673-678, 2004.

[4] Viola, P., Jones, M.J.: Robust Real-Time Face Detection. Int. J. Comput. Vision 57, 137-154 (2004)

[5] A. Hertzmann, C. E. Jacobs, N. Oliver, B. Curless, and D. H.Salesin. Image analogies. In Proc. ACM SIGGRAPH, 2001.

[6] Lienhart, R.: Stump-based 20x20 gentle adaboost frontal face detector, http://www.lienhart.de/

[7] Hameed, S.: Stump-based 20x20 frontal eye detector, http://umich.edu/ shameem

[8] S. Milborrow and F. Nicolls. Locating facial features with an extended active shape model. In Proc. ECCV, 2008.

[9] Z. Farbman, R. Fattal, D. Lischinski, and R. Szeliski. Edge preserving decompositions for multi-scale tone and detailmanipulation. ACM Trans. Graphics, 27(3):110, 2008.

[10] N. Ojima, K. Yoshida, O. Osanai, and S. Akasaki. Image synthesis of cosmetic applied skin based on optical properties of foundation layers. In Proceedings of International Congress of Imaging Science, pages 467468, 1999.

[11]F. Nars. Makeup your Mind.PowerHouse Books, 2004.
[12] Y. Shan, Z. Liu, and Z. Zhang. Image-based surface detail transfer. In Proc. CVPR, 2001.

[13] Taaz.com. http://www.taaz.com/.

[14]C. Tomasi and R. Manduchi. Bilateral filtering for gray and color images. In Proc. ICCV, 1998.

[15] W.-S. Tong, C.-K. Tang, M. S. Brown, and Y.-Q. Xu. Example-based cosmetic transfer. In Proc. Pacific Conference on Computer Graphics and Applications, 2007.

[16] N. Tsumura, N. Ojima, K. Sato, M. Shiraishi, H. Shimizu,H. Nabeshima, S. Akazaki, K. Hori, and Y. Miyake. Imagebased skin color and texture analysis/synthesis by extracting hemoglobin and melanin information in the skin. ACMTrans. Graphics, 22(3):770-779, 2003.

[17] Imagenomic, Leesburg, VA, USA. Portraiture. (2014, Mar. http://imagenomic.com/pt.aspx

[18] Caltech, Pasadena, CA, USA. (1999). Face Database [Online]. Available:http://www.vision.caltech.edu/htmlfiles/

[19] X. Zhang, T. Sim, and X. Miao. Enhancing photographs with near infra-red images. In Proc. CVPR, 2008.

[20] N. Joshi, W. Matusik, E. H. Adelson, and D. J. Kriegman, "Personal photo enhancement using example images," ACM Trans. Graph., vol. 29,no. 2, pp. 1-15, 2010.

[21] M. Chai, L. Wang, Y. Weng, Y. Yu, B. Guo, and K. Zhou, "Single-view hair modeling for portrait manipulation," ACM Trans. Graph., vol. 31,no. 4, pp. 18, 2012.

[22] V. Blanz and T. Vetter, "A morphable model for the synthesis of 3Dfaces," in Proc. ACM SIGGRAPH, 1999, pp. 187-194.

[23] V. Blanz. (2003). Manipulation of Facial Attractiveness [Online].

Available:http://www.mpiinf.mpg.de/ blanz/data/attract iveness/

[24]C. Florea, A. Cap`aťa, M. Ciuc, and P. Corcoran, "Facial enhancement and beautification for HD video cameras," in Proc. IEEE Int. Conf.Consumer Electron., Jan. 2011, pp. 741-742.

[25] C.-W. Chen, D.-Y. Huang, and C.-S. Fuh, "Automatic skin color beautification,"in Arts and Technology. Berlin/Heidelberg, Germany: Springer-Verlag, pp. 157164, 2010.

[26] C. Lee, M. T. Schramm, M. Boutin, and J. P. Allebach, "An algorithm for automatic skin smoothing in digital portraits," in Proc. IEEE Int.Conf. Image Process., Nov. 2009, pp. 3149-3152.

[27] M. Ciuc, A. Capata, V. Mocanu, C. Florea, A. Pososin, and P. Corcoran, "Automatic face and skin beautification using face detection,” U.S.Patent 12512 843, Jul. 30, 2009.

[28] M. Ciuc, A. Capata, and C. Florea, "Eye beautification," U.S. Patent 2827 868, Jun. 30, 2010.

[29] D. Guo and T. Sim, "Digital face makeup by example," in Proc. CVPR,2009, pp. 1063-6919.

[30] P. Viola and M. J. Jones, "Robust real-time face detection," Int. J.Comput. Vision, vol. 57, no. 2, pp. 137-154, 2004. 
[31] T. F. Cootes, C. J. Taylor, D. H. Cooper, and J. Graham, "Active shape models: Their training and application," Comput. Vision ImageUnderstand., vol. 61, no. 1, pp. 38-59, 1995.

[32] Z. Farbman, R. Fattal, D. Lischinski, and R. Szeliski, "Edge-preserving decompositions for multi-scale tone and detail manipulation," ACMTrans. Graph., vol. 27, no. 3, pp. 67:1-67:10, Aug. 2008.

[33] Y. Eisenthal, G. Dror, and E. Ruppin, "Facial attractiveness: Beauty and the machine," Neural Computat., vol. 18, no. 1, pp. 119-142, 2006.

[34] T. Leyvand, D. Cohen-Or, G. Dror, and D. Lischinski, "Data-driven enhancement of facial attractiveness," in Proc. ACM SIGGRAPH, 2008,pp. 38:1-38:10.

[35] V. Coetzee, S. J. Faerber, J. M. Greeff, C. E. Lefevre, D. E. Re, and D. I. Perrett, "African perceptions of female attractiveness," PLoS One,vol. 7, no. 10, p. e48116, 2012.

[36] I. D. Stephen, M. J. L. Smith, M. R. Stirrat, and D. I. Perrett, "Facial skin coloration affects perceived health of human faces," Int. J. Primatol.,vol. 30, no. 6, pp. 845-857, 2009.

[37] Z. Farbman, R. Fattal, and D. Lischinski, "Diffusion maps for edge-aware image editing," in Proc. ACM SIGGRAPH Asia, 2010, pp. 145:1-145:10.

\section{Author Profile}

Priyanka Mehra received the B.E. degree in Instrumentation \& Control from IITM College RGPV, University Bhopal, M.P., India, in 2007.Currently, she is a pursuing Master of Engineering from Imperial college of Engineering and Research, Wagholi, Pune, Maharashtra SPPU University. Her current research interests include,"Facial Skin Embellishment Using Flexible Region Aware Mask. 